\title{
A search for gamma-ray emission from the Galactic plane in the longitude range between $37^{\circ}$ and $43^{\circ}$
}

\author{
F. A. Aharonian ${ }^{1}$, A. G. Akhperjanian ${ }^{7}$, J. A. Barrio ${ }^{2,3}$, K. Bernlöhr ${ }^{1}$, O. Bolz ${ }^{1}$, H. Börst ${ }^{5}$, H. Bojahr $^{6}$,
} J. L. Contreras ${ }^{3}$, J. Cortina ${ }^{2}$, S. Denninghoff ${ }^{2}$, V. Fonseca ${ }^{3}$, J. C. Gonzalez ${ }^{3}$, N. Götting ${ }^{4}$,

G. Heinzelmann ${ }^{4}$, G. Hermann ${ }^{1}$, A. Heusler ${ }^{1}$, W. Hofmann ${ }^{1}$, D. Horns ${ }^{4}$, A. Ibarra ${ }^{3}$, C. Iserlohe ${ }^{6}$, I. Jung ${ }^{1}$, R. Kankanyan ${ }^{1,7}$, M. Kestel ${ }^{2}$, J. Kettler ${ }^{1}$, A. Kohnle ${ }^{1}$, A. Konopelko ${ }^{1}$, H. Kornmeyer ${ }^{2}$, D. Kranich ${ }^{2}$, H. Krawczynski ${ }^{1}, \%$, H. Lampeitl ${ }^{1}$, E. Lorenz ${ }^{2}$, F. Lucarelli ${ }^{3}$, N. Magnussen ${ }^{6}$, O. Mang ${ }^{5}$, H. Meyer ${ }^{6}$, R. Mirzoyan ${ }^{2}$, A. Moralejo ${ }^{3}$, L. Padilla ${ }^{3}$, M. Panter ${ }^{1}$, R. Plaga ${ }^{2}$, A. Plyasheshnikov ${ }^{1, \S}$, J. Prahl $^{4}$, G. Pühlhofer ${ }^{1}$, W. Rhode ${ }^{6}$, A. Röhring ${ }^{4}$, G. P. Rowell ${ }^{1}$, V. Sahakian ${ }^{7}$, M. Samorski ${ }^{5}$, M. Schilling ${ }^{5}$, F. Schröder ${ }^{6}$, M. Siems ${ }^{5}$, W. Stamm ${ }^{5}$, M. Tluczykont ${ }^{4}$, H. J. Völk ${ }^{1}$, C. A. Wiedner ${ }^{1}$, and W. Wittek ${ }^{2}$

\footnotetext{
1 Max-Planck-Institut für Kernphysik, Postfach 103980, 69029 Heidelberg, Germany

2 Max-Planck-Institut für Physik, Föhringer Ring 6, 80805 München, Germany

3 Universidad Complutense, Facultad de Ciencias Físicas, Ciudad Universitaria, 28040 Madrid, Spain

4 Universität Hamburg, II. Institut für Experimentalphysik, Luruper Chaussee 149, 22761 Hamburg, Germany

${ }^{5}$ Universität Kiel, Institut für Experimentelle und Angewandte Physik, Leibnizstraße 15-19, 24118 Kiel, Germany

${ }^{6}$ Universität Wuppertal, Fachbereich Physik, Gaußstr. 20, 42097 Wuppertal, Germany

7 Yerevan Physics Institute, Alikhanian Br. 2, 375036 Yerevan, Armenia

$\S$ On leave from Altai State University, Dimitrov Street 66, 656099 Barnaul, Russia

\% Now at Yale University, PO Box 208101, New Haven, CT 06520-8101, USA
}

Received 4 January 2001 / Accepted 18 June 2001

\begin{abstract}
Using the HEGRA system of imaging atmospheric Cherenkov telescopes, a region of the Galactic plane $\left(-10^{\circ}<b<5^{\circ}, 38^{\circ}<l<43^{\circ}\right)$ was surveyed for $\mathrm{TeV}$ gamma-ray emission, both from point sources and of diffuse nature. The region covered includes 15 known pulsars, 6 known supernova remnants (SNR) and one unidentified EGRET source. No evidence for emission from point sources was detected; upper limits are typically below $0.1 \mathrm{Crab}$ units for the flux above $1 \mathrm{TeV}$. For the diffuse gamma-ray flux from the Galactic plane, an upper limit of $6.1 \times 10^{-15} \mathrm{ph} \mathrm{cm}^{-2} \mathrm{~s}^{-1} \mathrm{sr}^{-1} \mathrm{MeV}^{-1}$ was derived under the assumption that the spatial distribution measured by the EGRET instrument extends to the TeV regime. This upper flux limit is a factor of about 1.5 larger than the flux expected from the ensemble of gamma-ray unresolved Galactic cosmic ray sources.
\end{abstract}

Key words. gamma-rays: observations - ISM: cosmic rays

\section{Introduction}

Systems of imaging atmospheric Cherenkov telescopes, such as the HEGRA stereoscopic telescope system (Daum et al. 1997; Konopelko et al. 1999a), allow one to reconstruct the directions of air showers over the full field of view - with a radius of about $2^{\circ}$ - and can therefore be used for sky surveys (Pühlhofer et al. 1999). Here, we report on a survey of a rectangular patch of the sky of roughly $80 \mathrm{deg}^{2}$, centered on the Galactic plane at longitude $40^{\circ}$. The motivation for this survey was twofold:

- Search for diffuse gamma-ray emission from the Galactic plane;

- Search for gamma-ray point sources.

Send offprint requests to: H. Lampeitl, e-mail: lampeitl@daniel.mpi-hd.mpg.de
Diffuse emission from the Galactic plane results from the interactions of charged cosmic rays with interstellar gas confined to the plane or with photons. Diffuse emission in the energy range from tens of $\mathrm{MeV}$ to tens of $\mathrm{GeV}$ has been studied intensively by SAS 2 (Fichtel et al. 1975; Hartmann et al. 1979), COS B (Mayer-Hasselwander et al. 1980, 1982) and EGRET (Hunter et al. 1997). The basic features can be modeled assuming $\pi^{\circ}$ decay as the dominant mechanism, with the gamma-ray emission proportional to the product of the gas column density and the cosmic-ray density (see, e.g., Hunter et al. 1997; Fichtel et al. 1975; Strong et al. 1988; Bloemen 1989; Bertsch et al. 1993). The distribution of cosmic rays is assumed to follow the matter density with a characteristic correlation scale of 1.5 to $2 \mathrm{kpc}$ (Hunter et al. 1997). In addition to diffuse Galactic gamma rays, there is also a small extragalactic component, which should be fairly isotropic 
(Fichtel et al. 1978; Sreekumar et al. 1998). Above $1 \mathrm{GeV}$, data show an excess in gamma-ray flux over model predictions (Hunter et al. 1997; see however Aharonian \& Atoyan 2000). At these energies, contributions from inverse Compton scattering of electrons start to become relevant (see, e.g., Porter \& Protheroe 1997). In response, revised models speculate that the local measurements of the electron flux may not be representative for the entire Galaxy (Porter \& Protheroe 1997; Pohl \& Esposito 1998). Electron propagation is limited by radiative losses, and the local electron spectra are strongly influenced by the history of sources in the local neighborhood (Aharonian et al. 1995; Pohl \& Esposito 1998). In case that the solar system is in an "electron void", diffuse gamma-ray emission at high energies could be an order of magnitude above predictions based on local electron spectra (Pohl \& Esposito 1998). Detailed models of the full spectrum of diffuse gamma-ray emission from the Galaxy (Moskalenko \& Strong 1999, 2000; Strong et al. 2000) also favour a harder electron spectrum. In the Galactic plane another "diffuse" gamma-ray flux component arises from the hard energy spectrum of those Galactic CRs that are still confined in the ensemble of their unresolved sources. Assuming these to be SNRs, Berezhko \& Völk (2000) showed that their spatially averaged contribution to the diffuse gamma-ray flux at $1 \mathrm{TeV}$ should exceed the model predictions of Hunter et al. (1997) by almost an order of magnitude. It is therefore of significant interest to search for the extension of the diffuse emission from the Galactic plane at higher energies. Upper limits on diffuse gamma-ray emission at $\mathrm{TeV}$ energies have been reported by Reynolds et al. (1993), and LeBohec et al. (2000), at a level of a few $10^{-3}$ of the cosmic-ray flux. The most stringent limits at higher energies, above $100 \mathrm{TeV}$, were given by Borione et al. (1997), and constrain the diffuse flux to less than $3 \times 10^{-4}$ of the cosmic-ray flux.

The Galactic plane is also a region rich in potential gamma-ray point sources. For obvious reasons, supernova remnants as well as pulsar driven nebulae cluster along the Galactic plane. Both types of objects are almost certainly accelerators of cosmic rays and emitters of highenergy gamma radiation. Theoretical models predict that typical gamma-ray fluxes from the majority of these objects are below the detection threshold of the current generation of instruments (see, e.g., Aharonian et al. 1997; Drury et al. 1994). However, both the lack of knowledge of the individual source parameters as well as approximations used in the modeling result in large uncertainties in the predictions for individual objects, by an order of magnitude or more. In addition to pulsars and supernova remnants, many unidentified EGRET sources lie in the Galactic plane. Given the density of source objects, a survey provides an efficient way to search for gamma-ray emission.

The selection of the region to be surveyed was governed both by astrophysical and by practical considerations. Using the EGRET observations of the diffuse gamma-ray flux (Hunter et al. 1997) as a guideline, a region within the inner Galaxy $\left(|l|<40^{\circ}\right)$ and close to the Galactic equator should show the strongest emission, corresponding to the gas column density. Similarly, the density of supernova remnants (Green 1998) and pulsars (Tayler 1993) is highest in this region. However, from the location of the HEGRA telescope system, at $28^{\circ} 45^{\prime} \mathrm{N}$, the Galactic centre region can only be observed at rather unfavourable zenith angles of $60^{\circ}$ or more, and the observation time is rather limited. At such large zenith angles, the energy threshold of the telescopes is increased from $500 \mathrm{GeV}$ for vertical showers to $5 \mathrm{TeV}$ at $60^{\circ}$ (Konopelko et al. 1999b). Best sensitivity is obtained for observations at zenith angles below $30^{\circ}$, favouring regions at larger Galactic longitude. Another potential problem for Cherenkov observations is the large and spatially varying background light from regions of the Galactic plane, which may result in non-uniform sensitivity across the field of view of the Cherenkov telescopes.

As a result of these considerations, a rather dark region at about $40^{\circ}$ longitude was chosen for the survey, with an extension in Galactic longitude of $5^{\circ}$ and in latitude of $15^{\circ}$ (Fig. 2). The relatively large range in latitude was chosen in order to cover the full range expected for diffuse gamma-ray emission, taking into account that the inverse Compton mechanisms may result in a wider latitude distribution than observed in the EGRET data, and to include additional background regions at large latitude. The observation region includes part of the "Sagittarius Arm", one of the spiral arms of the Galaxy. In addition, it hosts 15 known pulsars (Table 1), 6 supernova remnants (Table 2), and the unidentified EGRET source 3EG J1903+0550 (Hartman et al. 1999). There are no bright stars in the survey region, which might seriously impact the observations; the brightest star is of $m_{v}=4.3^{\mathrm{mag}}$, and there are 10 stars brighter than $6^{\mathrm{mag}}$. (A $4.3^{\mathrm{mag}}$ star increases the DC current in a camera pixel from 0.8 to $10 \mu \mathrm{A}$, resulting in an increased noise of 1.5 ph.e. rms).

\section{The HEGRA IACT system}

The HEGRA stereoscopic system (Daum et al. 1997; Konopelko et al. 1999a) of imaging atmospheric Cherenkov telescopes (IACTs) is located on the Canary Island of La Palma, on the site of the Observatorio del Roque de los Muchachos, at $28^{\circ} 45^{\prime} \mathrm{N}, 17^{\circ} 53^{\prime} \mathrm{W}$, $2200 \mathrm{~m}$ a.s.l. The stereoscopic telescope system comprises of five telescopes (CT2-CT6). One additional telescope (CT1) is operated in stand-alone mode. The system telescopes are arranged on the corners and in the centre of a square with $100 \mathrm{~m}$ side length. Each of them is equipped with tessalated $8.5 \mathrm{~m}^{2}$ mirrors of $5 \mathrm{~m}$ focal length, and a camera with 271 photomultiplier pixels in the focal plane. The field of view of each camera has $4.3^{\circ}$ diameter; the pixel diameter corresponds to $0.25^{\circ}$. The telescope system is triggered when in at least two cameras two neighboring pixels show a signal above $6-8$ photoelectrons. Signals from the cameras are recorded using a $120 \mathrm{MHz}$ Flash-ADC system, which is read out after a trigger. 


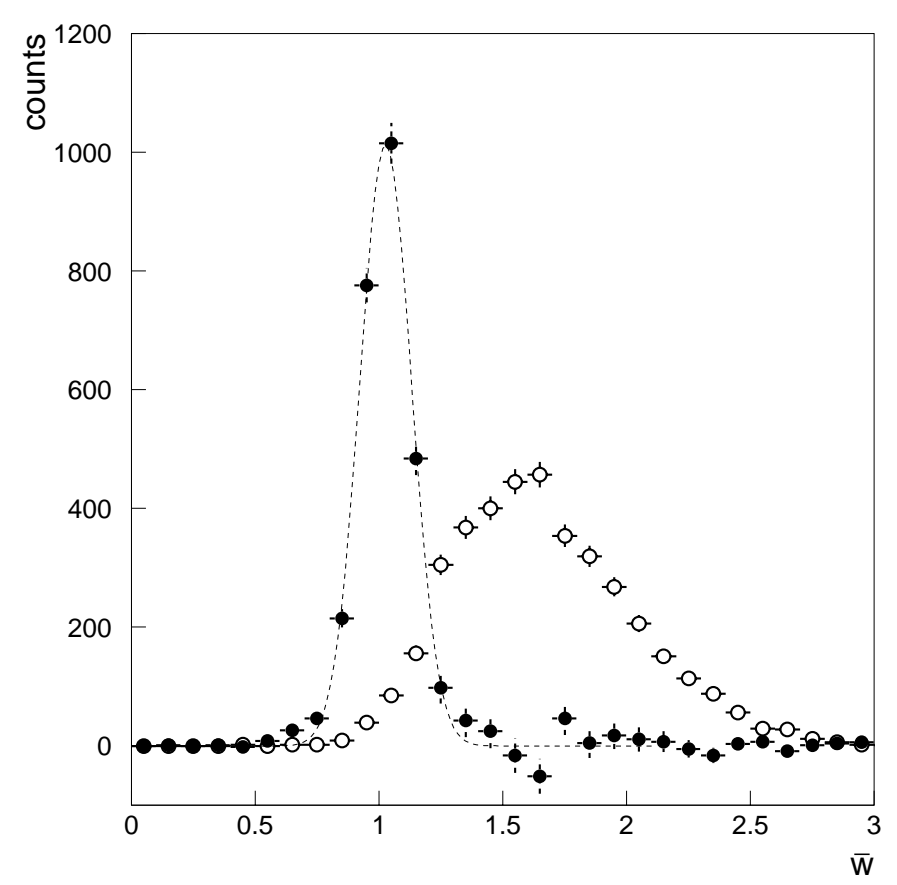

Fig. 1. Mean scaled width $\bar{w}$ of Cherenkov images in events where at least four telescopes triggered, for $(\bullet)$ gamma-rays from the Crab reference sample, and for (o) cosmic-ray showers.

Details of the camera hardware and of the trigger system are given in Hermann (1995) and Bulian et al. (1998). The pointing of the telescopes is known better than 1 arcmin. (Pühlhofer et al. 1997). On the basis of the stereoscopic analysis of Cherenkov images, shower directions can be reconstructed with a precision of $0.1^{\circ}$, and shower energies with a resolution of $20 \%$ or better (Daum et al. 1997; Aharonian et al. 1999a,b). For vertical incidence of showers, the energy threshold is $500 \mathrm{GeV}$ for gamma-rays, and increases to $0.9 \mathrm{TeV}$ at $30^{\circ}$ and $1.8 \mathrm{TeV}$ at $45^{\circ}$ (Konopelko et al. 1999b). Cosmic ray showers are suppressed exploiting the width of Cherenkov images. A "mean scaled width" $\bar{w}$ is defined by scaling the observed widths to the expected width for gamma-ray images, which depends on the intensity of the images and the distance to the shower core, and averaging over telescopes. Gamma-rays cause a peak in $\bar{w}$ at 1 , with a Gaussian width of about 0.1 to 0.12 (Fig. 1). Nucleonic showers have larger $\bar{w}$ values, peaking around 1.7. While more sophisticated identification schemes (e.g., Daum et al. 1997) can reach slightly better sensitivity, the default (and most stable) analysis schemes are based on cuts in $\bar{w}(\bar{w}<1.1 \ldots 1.3)$, combined with an angular cut relative to the source of about $0.1^{\circ}$.

\section{The data set}

The data used in this survey were taken over 37 days in June, July and August 1999 with the complete 5-telescope system.

As illustrated in Fig. 2, the total observation time of $88 \mathrm{~h}$ was distributed between three scans in Galactic latitude, centered at Galactic longitude of $39^{\circ}, 40.5^{\circ}$ and $42^{\circ}$, chosen to guarantee an overlap of the effective fields of view for the three scans (the scan positions given here and below refer to the center of the field of view). Each scan was conducted in $1^{\circ}$ steps in latitude between $-4^{\circ}$ and $+4^{\circ}$, with an additional control region at $-9^{\circ}$. The typical time per scan point was about $15 \mathrm{~min}$ per day, and the scans were repeated on several days. The points at latitude $0^{\circ}$ received twice the exposure, once covered going from $+4^{\circ}$ to $0^{\circ}$ and once going from $-4^{\circ}$ to $0^{\circ}$. A quality selection of the data sets was based primarily on the average trigger rate of the telescope system. A fraction of the data set suffered from extinction of Cherenkov light due to Sahara dust in the atmosphere (the so-called Calima, a well-known phenomenon at the Canary Islands). The remaining data set is quite uniform in detection rate and not affected by Calima. In total it encompasses $41.7 \mathrm{~h}, 18.6 \mathrm{~h}$ for the latitude scans at $39^{\circ}$ longitude, $14.6 \mathrm{~h}$ at $40.5^{\circ}$ longitude and $8.5 \mathrm{~h}$ at $42^{\circ}$ longitude. The data cover zenith angles between $21^{\circ}$ and $35^{\circ}$; the zenith angle of a run and the Galactic latitude are somewhat correlated, with data at $4^{\circ}$ latitude covering zenith angles between $20^{\circ}$ and $28^{\circ}$, compared to $28^{\circ}$ to $35^{\circ}$ for the runs at $-9^{\circ}$ latitude. In total, $1.4 \times 10^{6}$ Cherenkov events are used.

The analysis of Cherenkov images could potentially suffer from variations in the sky brightness over the scan region. Since the readout electronics of the telescopes is $\mathrm{AC}$ coupled, a star illuminating a pixel will not cause baseline shifts, but it will still result in increased noise in that pixel. As a measure of the influence of night sky background light, the baseline noise of the photomultiplier signal was determined. The RMS of the baseline noise of the individual PMTs is quite homogeneous over the region and amounts in average to about $1 \mathrm{ADC}$ count. The brightest stars in the region increase the RMS to $1.5 \mathrm{ADC}$ counts; this is uncritical for further image analysis.

For reference and comparison, observations of the Crab Nebula in the winters 1997/1998, 1998/1999 and $1999 / 2000$ were used, and were subject to identical selection criteria. In total, $41.7 \mathrm{~h}$ of observations at zenith angles between $18^{\circ}$ and $32^{\circ}$ were used, with a roughly uniform coverage of the zenith angle range.

The last telescope integrated into the system, CT2, one of the corner telescopes, was only used for part of the Crab reference data sets. Therefore, all analyses discussed in the following were performed both using only the four telescopes, as well as the full set of five telescopes. Because of the good agreement between the simulations and the measured rate with the complete system (see below), the five-telescope limits are quoted as the final results.

\section{Search for gamma-ray point sources}

The cuts on the telescope images and the shower reconstruction follow earlier work. In particular, only images with at least 40 photoelectrons are accepted and the centroid of the image has to be within $1.7^{\circ}$ from the camera centre, in order to exclude truncated images. Showers with 


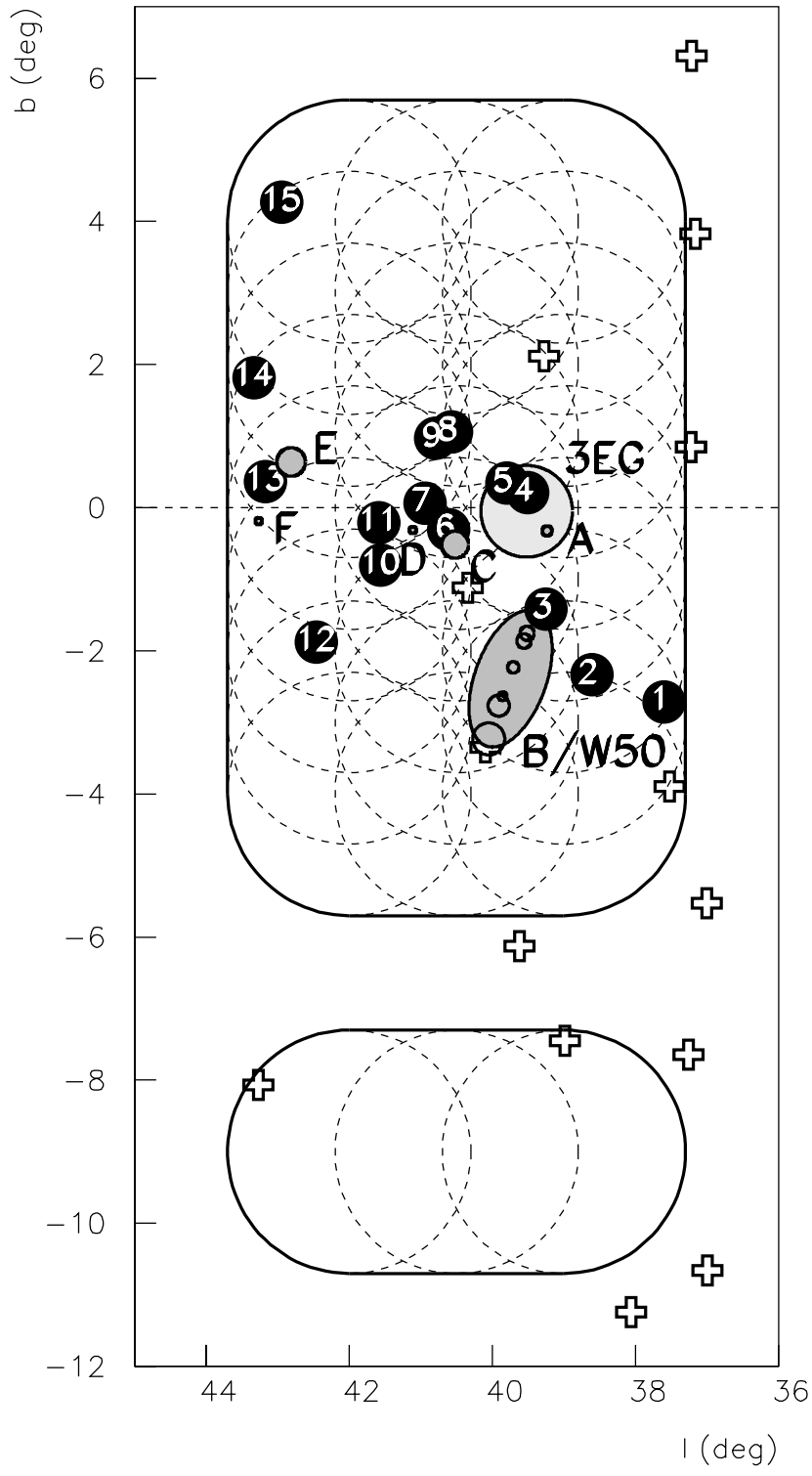

Fig. 2. Borders of the survey region (solid lines), and objects in this region. The borders are shown for an effective field of view of $1.7^{\circ}$ radius, corresponding to the radius where the detection efficiency is half of that compared to the value on the optical axis of the telescopes. Individual scan points are indicated as dashed circles. Pulsars are shown as black circles, supernova remnants as shaded regions, and stars brighter than $6^{\text {mag }}$ as crosses. The numbers of pulsars and SNR correspond to Tables 1 and 2. The light gray area indicates the position of the EGRET source 3EG J1903+0550 and the $1 \sigma$ error box.

reconstructed cores up to $300 \mathrm{~m}$ from the center telescope are accepted.

The performance of the telescope system for offaxis gamma-rays was investigated in detail using Monte Carlo simulations, for the zenith angle range in question. Figure 3 shows, as a function of shower inclination relative to the telescope axis, the rate at which showers are triggered and reconstructed with all five telescopes for a Crab-like source. The telescope system shows a FWHM
Table 1. Known pulsars in the survey region. Columns include the Galactic longitude $l$ and latitude $b$, the period $P$ and the period derivative $\dot{P}$. The dispersion measure (DM) can be used to estimate the distance to the pulsar, assuming a uniform density of thermal electrons in the Galaxy, in the order of $0.1-$ $0.01 \mathrm{~cm}^{-3}$ and dividing the DM by this number. Data taken from Tayler (1993).

\begin{tabular}{|r|l|c|c|c|c|c|}
\hline & $\begin{array}{l}\text { Name PSR } \\
\mathrm{J}(2000)\end{array}$ & $l\left[^{\circ}\right]$ & $b\left[^{\circ}\right]$ & $P[\mathrm{~s}]$ & $\begin{array}{c}\dot{P} \\
{\left[10^{-15}\right]}\end{array}$ & $\begin{array}{c}D M \\
{\left[\mathrm{~cm}^{-3} \mathrm{pc}\right]}\end{array}$ \\
\hline 1 & $1909+0254$ & 37.6 & -2.7 & 0.99 & 5.5287 & 172.1 \\
2 & $1910+0358$ & 38.6 & -2.3 & 2.33 & 4.53 & 78.8 \\
3 & $1908+04$ & 39.2 & -1.4 & 0.29 & - & 217 \\
4 & $1902+0556$ & 39.5 & +0.2 & 0.75 & 12.896 & 179.7 \\
5 & $1902+06$ & 39.8 & +0.3 & 0.67 & - & 530 \\
6 & $1906+0641$ & 40.6 & -0.3 & 0.27 & 2.1352 & 473 \\
7 & $1905+0709$ & 40.9 & +0.1 & 0.65 & 4.92 & 269 \\
8 & $1901+0716$ & 40.6 & +1.1 & 0.64 & 2.40 & 253 \\
9 & $1902+07$ & 40.8 & +1.0 & 0.49 & - & 90 \\
10 & $1910+07$ & 41.6 & -0.8 & 2.71 & - & 115 \\
11 & $1908+07$ & 41.6 & -0.2 & 0.21 & - & 10 \\
12 & $1915+07$ & 42.5 & -1.9 & 1.54 & - & 50 \\
13 & $1908+0916$ & 43.2 & +0.4 & 0.83 & 0.098 & 250 \\
14 & $1904+10$ & 43.3 & +1.8 & 1.86 & - & 140 \\
15 & $1854+10$ & 42.9 & +4.3 & 0.57 & & 250 \\
\hline
\end{tabular}

Table 2. Known supernova remnants in the survey region (Green 1998). Most are shell-type SNR. For W50 individual points known as X-ray emitters are given (see e.g. Safi-Harb et al. 1997). For the unidentified EGRET source the location and $1 \sigma$ error box are given (Hartmann et al. 1999).

\begin{tabular}{|c|c|c|c|c|}
\hline & Name & $l\left[^{\circ}\right]$ & $b\left[^{\circ}\right]$ & diameter $\left[^{\circ}\right.$ \\
\hline $\mathrm{A}$ & $3 \mathrm{C} 396$ & 39.2 & -0.3 & $\sim 0.13$ \\
\hline \multirow[t]{6}{*}{$\mathrm{B}$} & W50 & 39.7 & -2.0 & $\sim 2 \times 1$ \\
\hline & SS-433 & 39.71 & -2.25 & \\
\hline & e1 & 39.75 & -2.66 & $\sim 0.12$ \\
\hline & $\mathrm{e} 2$ & 39.91 & -2.80 & $\sim 0.3$ \\
\hline & e3 & 40.09 & -3.21 & $\sim 0.45$ \\
\hline & $\mathrm{w} 2$ & 39.51 & -1.76 & \\
\hline $\mathrm{C}$ & - & 40.5 & -0.5 & $\sim 0.4$ \\
\hline $\mathrm{D}$ & 3C 397 & 41.1 & -0.3 & $<0.1$ \\
\hline $\mathrm{E}$ & - & 42.8 & +0.6 & 0.4 \\
\hline $\mathrm{F}$ & W49B & 43.3 & -0.2 & $<0.1$ \\
\hline $3 \mathrm{EG}$ & 3EG J1903+0550 & 39.52 & -0.05 & $1 \sigma 0.64$ \\
\hline
\end{tabular}

field-of-view of about $3.5^{\circ}$. Also shown is the actual Crab rate derived from $4.1 \mathrm{~h}$ of observations. During pointed observations with the HEGRA IACT system, the source is usually positioned $0.5^{\circ}$ off-center. The angular resolution is, within $20 \%$, independent of the inclination of the shower axis and varies between about $0.11^{\circ}$ for events triggered by at least two of the five telescopes, to $0.07^{\circ}$ for five-telescope events. The angular resolution is measured by the Gaussian width of a 1-dimensional projected angular distribution, corresponding to the $40 \%$ containment 


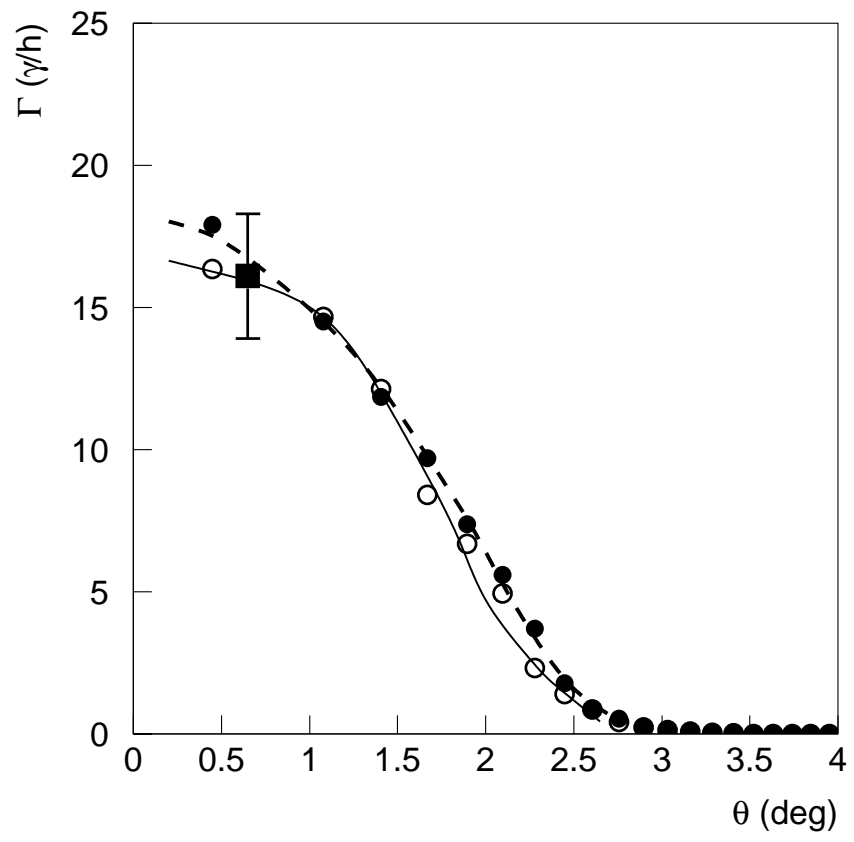

Fig. 3. Detection rates for a point source with the flux and spectrum of Crab Nebula, as a function of the angle of incidence $\Theta$ of the photons relative to the telescope axis. The simulations were performed for a five telescope system, and are shown for events where all telescopes trigger. The rates include a cut on the mean scaled width $<1.1$. Also shown is the rate measured for the Crab Nebula, positioned $0.5^{\circ}$ off-axis. Full symbols: $20^{\circ}$ zenith angle, open symbols: $30^{\circ}$.

radius $^{1}$ Since events with five triggered telescopes have both a better angular resolution and an improved hadron rejection compared to events with fewer telescopes, they contribute the bulk of the significance in the detection of sources. In fact, indiscriminate combination of 2,3 , 4 and 5-telescope events may deteriorate the sensitivity compared to 5-telescope events alone. For these reasons, the current analysis is based exclusively on events with five triggered telescopes for point sources and on four and five triggered telescopes for extended sources.

For a given point source candidate, events reconstructed within $0.11^{\circ}$ of the source direction were counted, after applying a cut on $\bar{w}$ at 1.1 to reject cosmic-ray events. In case of extended objects, such as SNR, the angular cut was increased to $0.2^{\circ}$ or $0.25^{\circ}$ depending on the source size, to ensure that the whole source is contained in the search region and in addition the 4-telescope events were used, since angular resolution is no longer critical. To estimate backgrounds, three regions of the same size as the source region were used, rotated by $90^{\circ}, 180^{\circ}$ and $270^{\circ}$ around the telescope axis, relative to the source. This background estimate can only be applied for sources more than $0.12^{\circ}$ away from the telescope axis (otherwise the source region and the background regions would overlap).

\footnotetext{
1 Non Gaussian tails depend on the number of telescopes used for reconstruction and are well below $10 \%$ for the 5 -telescope events.
}

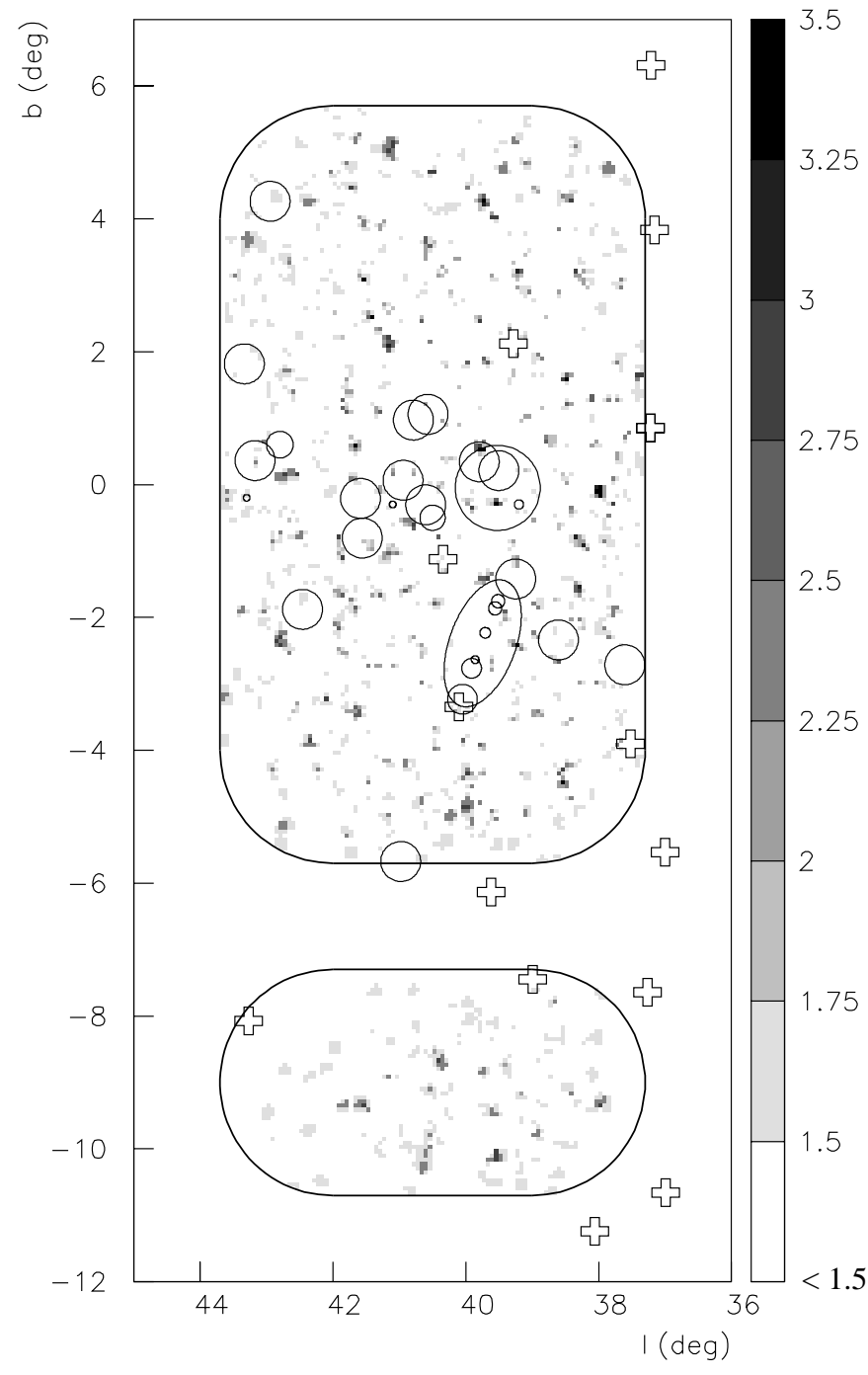

Fig. 4. Map of the significances. Only significances above 1.5 sigma are shown. Superimposed are the locations of potential sources and the borderline of the observed region (see also Fig. 2).

On-axis source regions are therefore excluded. A source region which is on-axis for one scan point will, of course, be off-axis for the neighbouring scan points, hence the net loss is small. The significance for a detection is then calculated according to Li \& Ma (1983), with $\alpha=1 / 3$ corresponding to the three background regions per source region.

In a first search for gamma-ray sources at arbitrary locations, significances were calculated for a twodimensional grid with $0.0625^{\circ}$ step size, covering the survey region. Note that the step size is smaller than the angular resolution to ensure that no sources are missed. This implies, however, that significances quoted for adjacent points are highly correlated. The highest significance observed is $4.1 \sigma$, compatible with the expected distribution in the absence of sources. The mean significance is 0.006 , the rms width of the distribution 1.027 , which shows that the technique used for background estimates is 


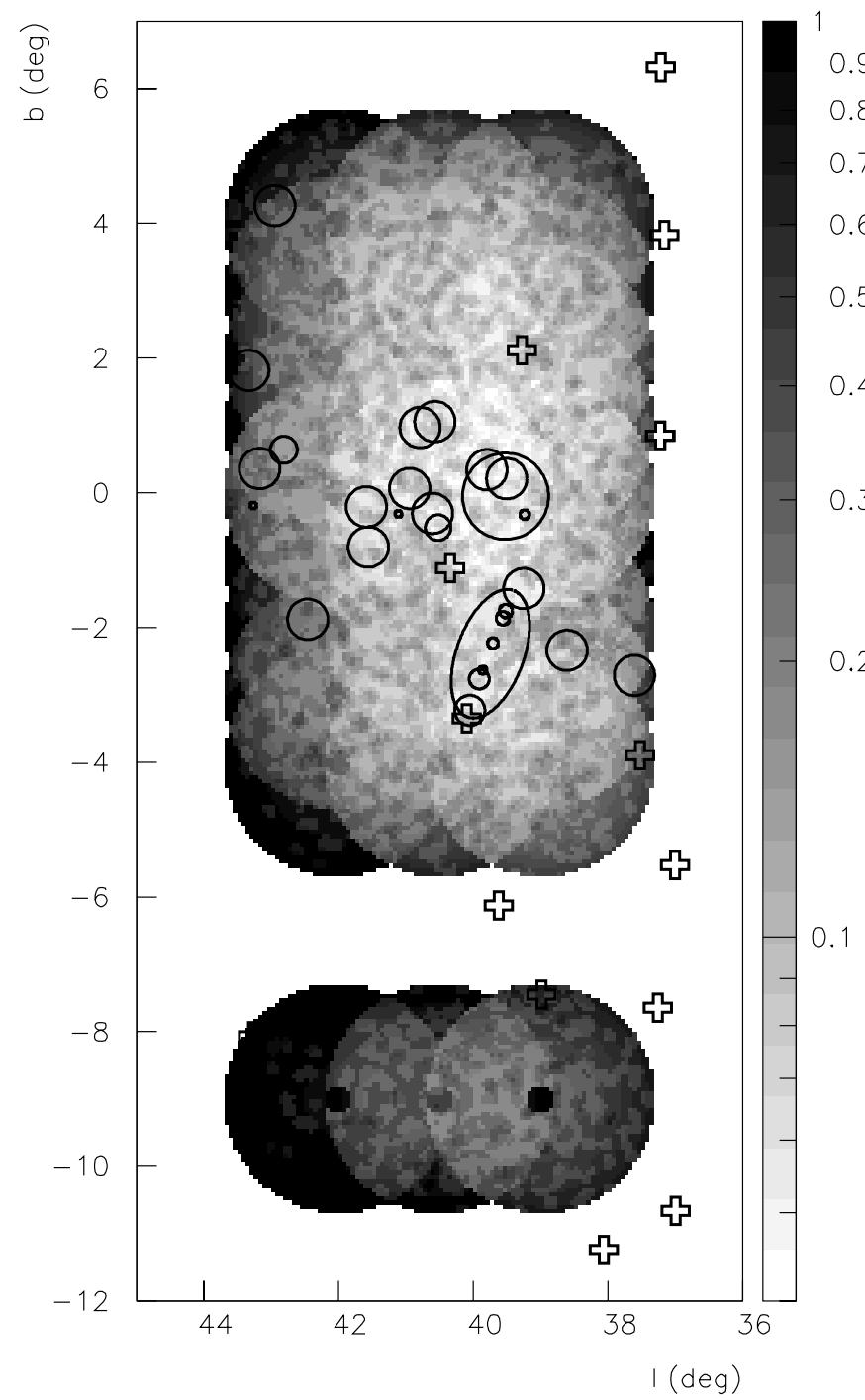

Fig. 5. Upper limits for the gamma-ray flux above $1 \mathrm{TeV}$, in units of the Crab flux. Upper limits above the flux of the Crab are shown in black.

sufficiently accurate. A significance map is shown in Fig. 4. The points with the highest significances do not correspond to locations of any known potential source as listed in Tables 1 and 2. Upper limits are calculated according to the procedure given in O. Helene (1983) and dividing this number by the expected number of events for a Crab like source seen at the same angle $\theta$ in the camera (see Fig. 3). To predict the expected event rate the HEGRA Monte Carlo code (Konopelko 1999a) were used to calculate the acceptance over the FoV. In the simulations the Crab spectrum is taken to be $\mathrm{d} \Phi / \mathrm{d} E=2.7( \pm 0.2 \pm 0.8) \times$ $10^{-11} E^{-2.59( \pm 0.06 \pm 0.1)} \mathrm{ph} \mathrm{cm}^{-2} \mathrm{~s}^{-1} \mathrm{Tev}^{-1}$ (Konopelko 1998). An additional scaling of the upper limit by $28 \%$ accounts for losses caused by the tight angular cut for point sources. The resulting upper limits are shown in Fig. 5. For the well-sampled points of the survey the limits are below $10 \%$ of the Crab flux. The limits given assume a slope of the energy spectrum similar to the Crab Nebula.
Table 3. Upper limits for specific objects. Columns show the observation time used in the analysis, the number of $\mathrm{ON}$ and OFF-source events (for a three times larger OFF region), the significance of detection and the $99 \%$ upper limit in units of the Crab flux. Objects are treated as point sources (type P) or extended sources (type E1: $\vartheta=0.2 \mathrm{deg}$, type E2: $\vartheta=0.25 \mathrm{deg}$ ).

\begin{tabular}{|l|c|c|c|c|c|c|}
\hline Object & Type & $T[\mathrm{~h}]$ & ON & OFF & $\sigma$ & $\phi^{99 \%}$ \\
\hline J1909+0254 & $\mathrm{P}$ & 3.87 & 0 & 2 & -1.07 & 0.150 \\
$\mathrm{~J} 1910+0358$ & $\mathrm{P}$ & 6.93 & 1 & 6 & -0.70 & 0.086 \\
$\mathrm{~J} 1908+04$ & $\mathrm{P}$ & 10.75 & 0 & 10 & -2.40 & 0.046 \\
$\mathrm{~J} 1902+0556$ & $\mathrm{P}$ & 11.94 & 2 & 4 & 0.46 & 0.059 \\
$\mathrm{~J} 1902+06$ & $\mathrm{P}$ & 11.94 & 3 & 11 & -0.31 & 0.060 \\
$\mathrm{~J} 1906+0641$ & $\mathrm{P}$ & 12.46 & 1 & 5 & -0.49 & 0.051 \\
J1905+0709 & $\mathrm{P}$ & 8.78 & 2 & 7 & -0.19 & 0.074 \\
J1901+0716 & $\mathrm{P}$ & 7.06 & 2 & 5 & 0.21 & 0.106 \\
J1902+07 & $\mathrm{P}$ & 8.97 & 2 & 3 & 0.74 & 0.083 \\
J1910+07 & $\mathrm{P}$ & 8.40 & 2 & 6 & 0.00 & 0.087 \\
J1908+07 & $\mathrm{P}$ & 8.78 & 1 & 6 & -0.70 & 0.068 \\
J1915+07 & $\mathrm{P}$ & 1.99 & 0 & 2 & -1.07 & 0.214 \\
J1908+0916 & $\mathrm{P}$ & 2.70 & 2 & 0 & 2.35 & 0.321 \\
J1904+10 & $\mathrm{P}$ & 1.55 & 0 & 0 & 0.00 & 0.533 \\
J1854+10 & $\mathrm{P}$ & 1.80 & 0 & 1 & -0.76 & 0.285 \\
\hline SNR-3C396 & $\mathrm{P}$ & 12.31 & 4 & 6 & 1.04 & 0.082 \\
SNR-G40.5 & $\mathrm{E} 2$ & 9.62 & 30 & 108 & -0.90 & 0.062 \\
SNR-3C397 & $\mathrm{P}$ & 8.78 & 3 & 4 & 1.03 & 0.096 \\
SNR-G42.8 & $\mathrm{E} 2$ & 2.49 & 12 & 24 & 1.12 & 0.263 \\
SNR-W49B & $\mathrm{P}$ & 2.16 & 1 & 0 & 1.67 & 0.337 \\
\hline SS433 & $\mathrm{P}$ & 9.02 & 1 & 6 & -0.70 & 0.063 \\
SS433-e1 & $\mathrm{P}$ & 10.00 & 2 & 2 & 1.07 & 0.080 \\
SS433-e2 & E1 & 10.00 & 20 & 66 & -0.38 & 0.110 \\
SS433-e3 & E2 & 7.02 & 19 & 60 & -0.20 & 0.091 \\
SS433-w2 & $\mathrm{P}$ & 9.02 & 2 & 4 & 0.46 & 0.081 \\
\hline 3EG & E2 & 12.83 & 39 & 124 & -0.32 & 0.073 \\
\hline
\end{tabular}

However, since limits are given for an energy which corresponds to the peak differential detection rate, the variation of the limits with the assumed spectral index is small (Aharonian et al. 1995). For a variation of \pm 0.5 in the spectral index, the limits typically change by $6 \%$.

Specific flux limits were derived for the potential source candidates, namely the 15 pulsars, the 6 supernova remnants, and the EGRET source. In case of the fairly extended SNR W50, hot spots known from X-ray measurements were treated as point sources. The so called eastern lobe (e3, see Table 2) and the associated knot e2 were treated as extended sources. W50 has been observed previously by HEGRA and a more detailed analysis of the previous data set and a discussion of results on W50 will be given in a separate paper (Aharonian et al. 2001).

Table 3 lists the upper limits obtained for all objects. 


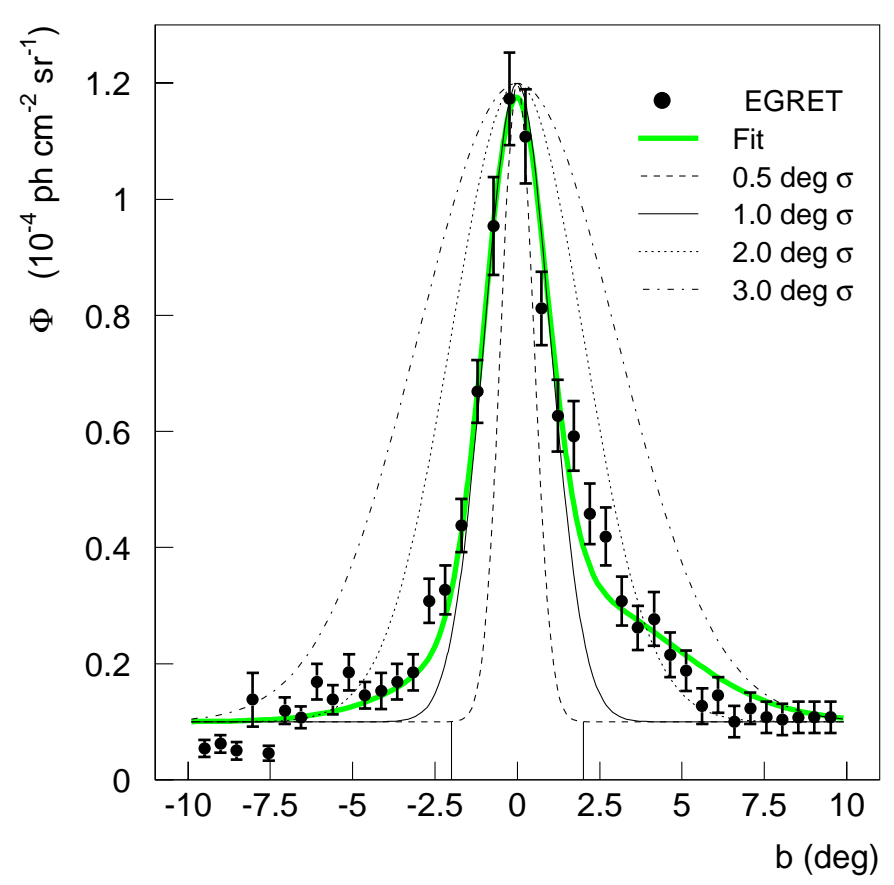

Fig. 6. Latitude dependence of the diffuse emission measured by EGRET for photon energies above $1 \mathrm{GeV}$, in the range of Galactic longitude $30^{\circ}$ to $50^{\circ}$, together with a fit by a sum of two Gaussians with widths and centres of $0.95^{\circ}, 3.16^{\circ}$ and $-0.05^{\circ}, 1.5^{\circ}$, respectively, and an intensity ratio of $4: 1$ in amplitude. A value of 0.1 was taken for the constant background. Data points taken from Hunter et al. (1997). In addition, Gaussian profiles with a $\sigma$ of $0.5^{\circ}, 1.0^{\circ}, 2.0^{\circ}$ and $3.0^{\circ}$ are shown.

\section{Search for diffuse gamma-ray emission from the Galactic plane}

Compared to the search for point sources, the search for diffuse gamma-ray emission from the Galactic plane is complicated by the extended structure of the emission region. The structure may be extended in latitude beyond the field of view. Figure 6 illustrates the profile in Galactic latitude as measured by EGRET in the longitude range $30^{\circ}$ to $50^{\circ}$, at energies above $1 \mathrm{GeV}$. The EGRET latitude profile can be represented as a sum of two Gaussians and an constant background value. Since the angular resolution of the EGRET instrument above $1 \mathrm{GeV}$ is narrower than the latitude extend of the diffuse emission the latitude dependence in Fig. 6 indicates real structure. Because of the possible change of the primary emission mechanism between the $\mathrm{GeV}$ and $\mathrm{TeV}$ range, from $\pi^{0}$ decay to inverse Compton scattering (Porter \& Protheroe 1997; Pohl \& Esposito 1998), and because of the completely different parent and target populations in the two cases, the latitude dependence may differ at $\mathrm{TeV}$ energies. At $\mathrm{GeV}$ energies, the latitude distribution of gamma-ray emissivity is characterized by the scale height of interstellar gas, around $100 \mathrm{pc}$ for atomic hydrogen (Lockman 1984) and somewhat less for molecular hydrogen (Dame 1987). In contrast, inverse-Compton interactions with the uniform microwave background photons are governed by the scale height of the electron component of cosmic rays, which might be characterized by the $\mathrm{kpc}$ scale describing the coupling of cosmic rays to matter. The scale height of farinfrared target photons, on the other hand, was given by Cox et al. (1986) as 100 pc, similar to the scale height of the gas. Porter \& Protheroe (1997) find, from numerical simulations of electron propagation, a scale height of more than $500 \mathrm{pc}$ for the inverse-Compton emissivity at $1 \mathrm{TeV}$.

In the following, we will discuss three techniques to search for diffuse gamma-ray emission. The techniques differ in the degree of assumptions they make concerning the latitude dependency of the diffuse emission, and also in their sensitivity to systematic variations in the performance and characteristics of the telescopes.

The most robust and model-independent - but also least sensitive - technique to derive limits on the diffuse flux simply selects events according to their shapes as gamma-ray candidates. A cut on $\bar{w}$ less than 1.0 keeps about $1 / 2$ of the gamma-rays, but rejects cosmic rays very efficiently (see Fig. 1). In order to achieve the best separation between gamma-ray images and cosmic-ray images, only five-telescope events were used. Such events with small $\bar{w}$ include genuine gamma-rays, electron showers from the diffuse cosmic-ray electron flux, and the tail of the distribution of cosmic-ray nuclear showers. Assigning all 428 events after cut as diffuse gamma-rays a $99 \%$ upper limit on the diffuse gamma-ray flux at $1 \mathrm{TeV}$ of $23.4 \times 10^{-15} \mathrm{ph} \mathrm{cm}^{-2} \mathrm{~s}^{-1} \mathrm{sr}^{-1} \mathrm{MeV}^{-1}$ results, for $|b|<5^{\circ}$. As mentioned in the discussion of the point source limits, the measurement determines the integral flux above the energy threshold of the telescope system, rather than directly determining the differential flux. Therefore the result depends in principle on the assumed spectral index. However, since flux values are quoted at energies corresponding roughly to the peak detection rates, the limits vary only very little with the spectral index. We note that the diffuse electron flux (see, e.g., the compilation by Barwick et al. 1998) should contribute about $1 / 3$ of the number of gamma-ray candidates; the limit can of course also be viewed as a limit on the electron flux, since the selection cuts are equally efficient for gamma-ray induced showers and electron-induced showers.

The limit obtained by this technique can be improved by subtracting, on a statistical basis, the number of gamma-ray candidate events resulting from cosmic-ray electrons or protons. Since it is virtually impossible to verify that simulations properly account for the tail towards very small $\bar{w}(<1.1)$ of the distribution of proton showers, such a subtraction has to be performed using an experimental background region, sufficiently far away from the Galactic plane such that diffuse gammaray emission from the plane is most probably negligible. Such a background data sample will only contain the isotropic electron and nucleon flux. In order to minimize instrumental effects, the background sample should be taken at the same time, and at identical zenith angles. Data sets can be normalized to each other using the rates 


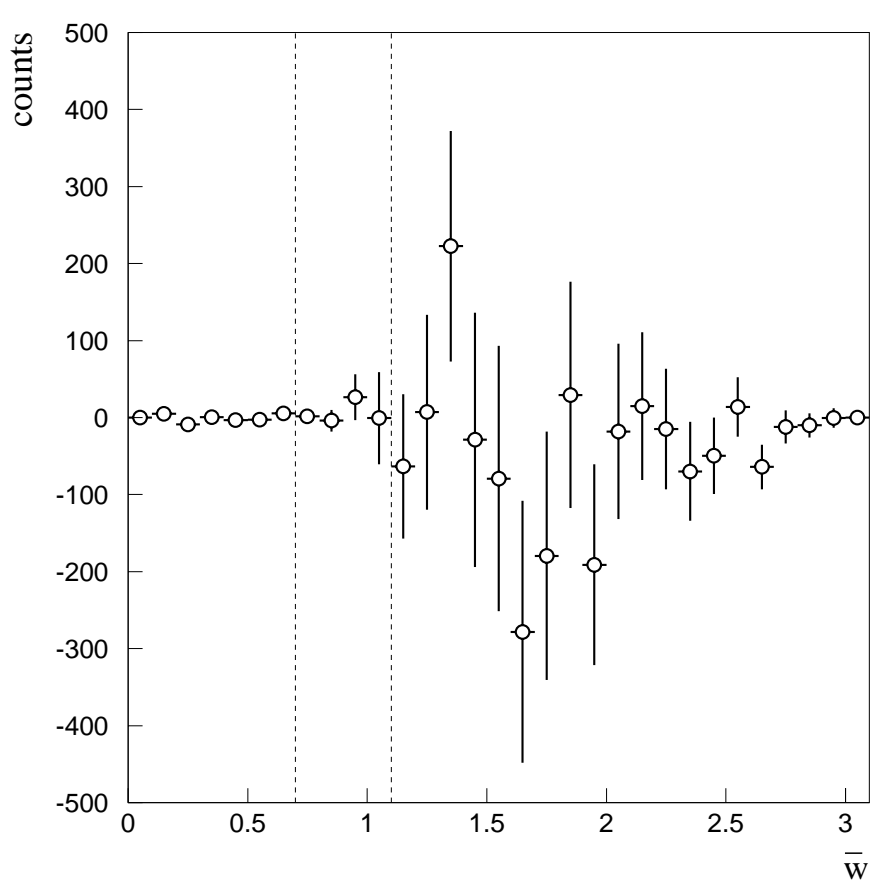

Fig. 7. Difference between the distributions in mean scaled width for the on-region $\left(|b|<2^{\circ}\right)$ and for the off-region $(|b|>$ $\left.2^{\circ}\right)$. The dashed lines indicate the expected gamma-ray region.

of events with large $\bar{w}(>1.4)$, well outside the gammaray region. Unfortunately, the availability of suitable background data samples with the same telescope configuration is limited to $4.1 \mathrm{~h}$ of data, and the statistical error of the background data set limits the sensitivity. After a $\bar{w}<1.1$ cut 1928 events survived compared to 141 events in the referenc sample. The scaling factor between the two datasets is determined to 13.3 and the MC simulations predict 555 events for a Crab like source smeared out over a FoV of $1.5^{\circ}$. After subtraction of isotropic components, we find a $99 \%$ limit on the diffuse flux in the region $|b|<5^{\circ}$ of $10.4 \times 10^{-15} \mathrm{ph} \mathrm{cm}^{-2} \mathrm{~s}^{-1} \mathrm{sr}^{-1} \mathrm{MeV}^{-1}$ at $1 \mathrm{TeV}$.

The final, and most sensitive analysis makes the assumption that diffuse gamma-ray emission from the Galactic plane is limited to the central parts of the scan region, and uses the outer parts of the scan region to estimate backgrounds. The range $|b|<2^{\circ}$ was considered the signal region, the range $|b|>2^{\circ}$ the background region. This cut is close to optimal for emission profiles with an rms width between $1^{\circ}$ and $2^{\circ}$ and results in a good balance in observation time $(\alpha \approx 1)$ between the $\mathrm{ON}$ and OFF regions. To ensure optimum quality of the events, only four- and five-telescope events were used, and the field of view was restricted to $1.5^{\circ}$ from the optical axis. A cut at 1.1 on the $\bar{w}$ was applied to reject cosmic-ray background $^{2}$. To account for a possible zenith-angle de-

\footnotetext{
2 The cuts on $\bar{w}$ differ between this and the first analysis; here, the goal is to optimize the significance of a weak signal $S$, e.g., the ratio $S / \sqrt{B}$, whereas analysis of the first type, where all backgrounds $B$ are counted as potential signal, need to optimize $S / B$.
}

pendence of background rates, data were grouped into four different ranges in zenith angle, $20^{\circ}-24^{\circ}, 24^{\circ}-28^{\circ}, 28^{\circ}-32^{\circ}$ and $32^{\circ}-36^{\circ}$. Also, the analysis was carried out separately for each scan band. For each range in zenith angle and each scan band, the expected number of events in the signal region was calculated based on the number of events observed in the corresponding areas of the camera for the background region. The expected and observed numbers of events were then added up for all zenith angles and scan bands. With a total number of 2387 gamma-ray events in the signal region, compared to 2353 expected events, there is no significant excess. As a cross check, Fig. 7 shows the background-subtracted distribution in $\bar{w}$ for events in the $|b|<2^{\circ}$ region. There is no indication of a significant excess in the gamma-ray region around a $\bar{w}$ of 1 indicated by the dashed lines. Also for larger values of $\bar{w}$, there is no significant excess or deficit, showing that background subtraction works properly.

In order to translate the limit in the number of events into a flux limit, one now has to make assumptions concerning the distribution in Galactic latitude of the diffuse radiation, since a spill-over of diffuse gamma-rays into the background region $|b|>2^{\circ}$ will effectively reduce the signal. For a profile with a width less or comparable to the EGRET profile - about $1^{\circ} \mathrm{rms}$ - a correction of $12 \%$ is applied and one finds a limit $6.1 \times$ $10^{-15} \mathrm{ph} \mathrm{cm}^{-2} \mathrm{~s}^{-1} \mathrm{sr}^{-1} \mathrm{MeV}^{-1}$ for the diffuse gammaray flux at $1 \mathrm{TeV}$, averaged over the $|b|<2^{\circ}$ region. The limit refers to an assumed spectral index of -2.6 , and changes by $+13 \%$ for an index of -2 , and by $-5 \%$ for an index of -3 . For wider distributions of $2^{\circ}$ and $3^{\circ} \mathrm{rms}$, the limit changes to $8.2 \times 10^{-15} \mathrm{ph} \mathrm{cm}^{-2} \mathrm{~s}^{-1} \mathrm{sr}^{-1} \mathrm{MeV}^{-1}$ and $12.8 \times 10^{-15} \mathrm{ph} \mathrm{cm}^{-2} \mathrm{~s}^{-1} \mathrm{sr}^{-1} \mathrm{MeV}^{-1}$, respectively. Figure 8 compares the upper limit with the extrapolation of the EGRET flux; also included are the limits by the Whipple group (Reynolds et al. 1993; LeBohec et al. 2000) and of the Tibet array (Amenomori 1997). Connecting the EGRET points with the HEGRA upper limit (and ignoring the highest-energy EGRET point with its large errors) one finds a lower limit of 2.5 on the spectral index of the diffuse gamma-ray emission.

Model predictions attempting to explain the excess flux in the $\mathrm{GeV}$ region by assuming an increased inverse Compton component (Porter \& Protheroe 1997; Pohl \& Esposito 1998), or by taking the contribution from unresolved SNRs into account (Berezhko \& Völk 2000), are given in the literature for different ranges in Galactic latitude $|b|$, and cannot be directly compared without assuming a latitude dependence of the diffuse flux. In Fig. 8 the solid line gives the model prediction of Berezhko \& Völk (2000) scaled by a factor of 3, taking into account the different latitude and longitude ranges used for the upper limit and the model and using the EGRET measurements at $20 \mathrm{GeV}$ as a guideline.

Even for pessimistic assumptions of a rather wide latitude dependence the model of Pohl \& Esposito (1998), if the inverse Compton flux is extrapolated from the $50 \mathrm{GeV}$ range discussed in the paper to the $\mathrm{TeV}$ range, exceeds the 


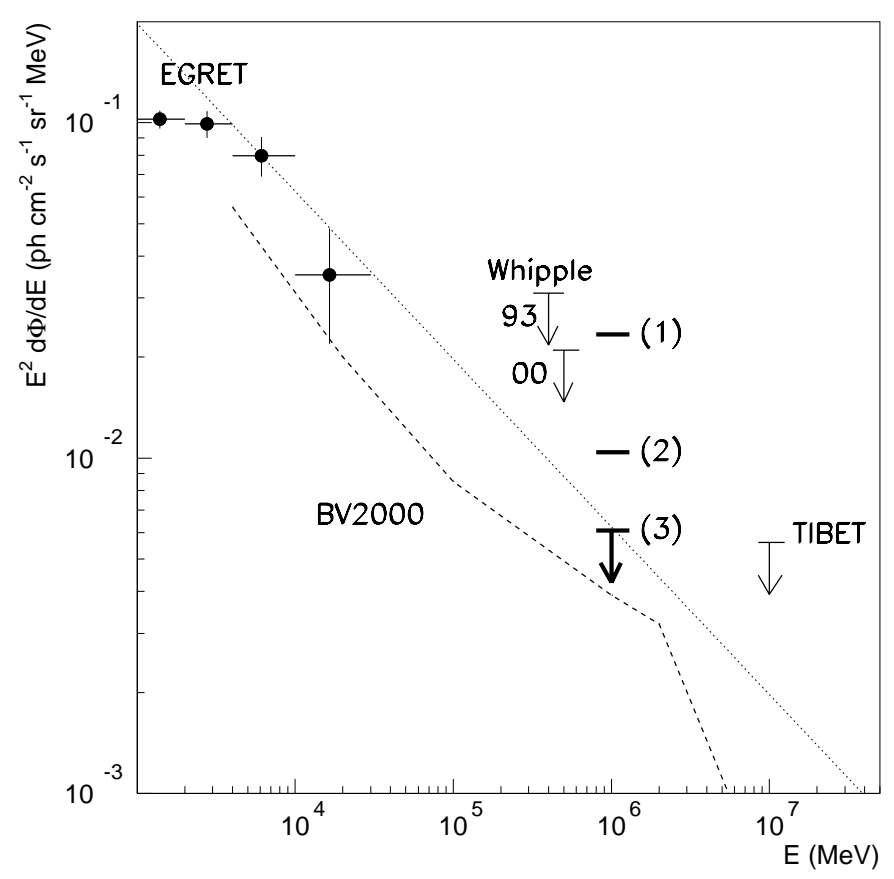

Fig. 8. Upper limits for the diffuse gamma-ray flux derived by this experiment. Assuming that all detected events are gammarays (1), using a independent data set for background subtraction (2), and using $|b|>2^{\circ}$ as background data and assuming the spatial distribution measured by the ERGRET instrument $\left(38^{\circ}<l<43^{\circ},|b|<2^{\circ}\right)(3)$. For detailed explanations see text. Also shown is the EGRET flux for $35^{\circ}<l<45^{\circ},|b|<2^{\circ}$, the Whipple upper limits for $38.5<l<41.5^{\circ},|b|<2^{\circ}$ (Reynolds et al. 1993; LeBohec 2000) and the Tibet upper limit (Amenomori 1997). The dotted line indicates an extrapolation of the EGRET data with an index of 2.5. The dashed line indicates the scaled "leaky box" model prediction by Berezhko \& Völk (2000) (BV2000) for the spatially averaged gammaray emission from the Galactic plane by those Galactic CRs that are still confined in their unresolved SNR sources, scaled to $|b|<2^{\circ}$.

HEGRA limit. At TeV energies, one is still sufficiently far from the Klein-Nishina regime such that the power-law extrapolation should be valid (Porter \& Protheroe 1997). Of course, a break in the electron injection spectrum could cause a corresponding break in the gamma spectrum between $50 \mathrm{GeV}$ and $1 \mathrm{TeV}$, and could be used to make the model consistent with the experimental limit.

\section{Concluding remarks}

A survey of the region $-10^{\circ}<b<5^{\circ}, 37^{\circ}<l<43^{\circ}$ near the Galactic plane did not yield evidence for $\mathrm{TeV}$ gammaray point sources, with typical flux limits of $10 \%$ of the Crab flux. In particular, 15 pulsars, 6 supernova remnants and one unidentified EGRET source were not detected as strong TeV sources.

A search for diffuse gamma-ray emission resulted in an upper limit of $6.1 \times 10^{-15} \mathrm{ph} \mathrm{cm}^{-2} \mathrm{~s}^{-1} \mathrm{sr}^{-1} \mathrm{MeV}^{-1}$ at $1 \mathrm{TeV}$, averaged over the region $38^{\circ}<l<43^{\circ},|b|<2^{\circ}$ and assuming the spatial emission profile measured by the EGRET instrument. Since the analysis used to derive this limit is only sensitive to the variation of the diffuse flux with $b$, rather than its absolute value, a distribution significantly wider than at EGRET energies will increase the limit. Other variants of the data analysis are sensitive to the absolute flux, but give less stringent limits of 23.4 and $10.4 \times 10^{-15} \mathrm{ph} \mathrm{cm}^{-2} \mathrm{~s}^{-1} \mathrm{sr}^{-1} \mathrm{MeV}^{-1}$. The limit on the $\mathrm{TeV}$ gamma-ray flux can be used to derive a lower limit on the spectral index of the diffuse radiation of 2.5, and to exclude models which predict a strong enhancement of the diffuse flux compared to conventional mechanisms. However the TeV flux limit is only about a factor of 1.5 larger than the predicted flux from unresolved SNRs. A more sensitive survey should therefore be able to test this prediction, together with a determination of the "diffuse" TeV gamma-ray spectrum that is directly related to the Galactic CR source spectrum.

Acknowledgements. The support of the HEGRA experiment by the German Ministry for Research and Technology BMBF and by the Spanish Research Council CYCIT is acknowledged. We are grateful to the Instituto de Astrofísica de Canarias for the use of the site and for providing excellent working conditions. We gratefully acknowledge the technical support staff of Heidelberg, Kiel, Munich, and Yerevan. GPR acknowledges receipt of a Humboldt Foundation postdoctoral fellowship.

\section{References}

Aharonian, F. A., Atoyan, A. M., \& Völk, H. J. 1995, A\&A, 294, L41

Aharonian, et al. 1995, J. Phys. G, 21, 419

Aharonian, F., et al. 1997, MNRAS, 291, 162

Aharonian, F., et al. 1999a, A\&A, 342, 69

Aharonian, F., et al. 1999b, A\&A, 349, 11

Aharonian, F. A., \& Atoyan, A. M. 2000, A\&A, 362, 937

Aharonian, F., et al. 2001, in preparation

Amenomori, M., et al. 1997, Proc. of the 25th ICRC, Durban, 3,117

Barwick, S. W., et al. 1998, ApJ, 498, 779

Berezhko, E. G., \& Völk, H. J. 2000, ApJ, 540, 923

Bertsch, D. L., et al. 1993, ApJ, 416, 587

Bloemen, J. B. G. M. 1989, ARA\&A, 27, 469

Borione, A., et al. 1997, ApJ, 493, 175

Bulian, N., et al. 1998, Astropart. Phys., 8, 223

Cox, P., Krügel, E., \& Metzger, P. G. 1986, A\&A, 63, 7

Dame, T. M., et al. 1987, ApJ, 322, 706

Daum, A., et al. 1997, Astropart. Phys., 8, 1

Drury, L. O., Aharonian, F. A., \& Völk, H. J. 1994, A\&A, 287, 959

Fichtel, C. E., et al. 1975, ApJ, 198, 163

Fichtel, C. E., Simpson, G. A., \& Thompson, D. J. 1978, ApJ, 222,833

Green, D. A. 1998, A Catalogue of Galactic Supernova Remnants, Mullard Radio Astronomy Observatory

Hartmann, R., et al. 1979, ApJ, 230, 597

Hartmann, R., et al. 1999, ApJS, 123, 79

Helene, O. 1983, NIM, 212, 319 
Hermann, G. 1995, Proc. of the Int. Workshop, Towards a Major Atmospheric Cherenkov Detector IV, Padua, ed. M. Cresti, 396

Hunter, et al. 1997, ApJ, 481, 205

Konopelko, A., et al. 1998, Proc. 16th ECRS, Alcala, Spain, ed. J. Medina, 523

Konopelko, A., et al. 1999a, Astropart. Phys., 10, 275

Konopelko, A., et al. 1999b, J. Phys. G, 25, 1989

LeBohec, S., et al. 2000, ApJ, 539, 209

Li, T., \& Ma, Y. 1983, ApJ, 272, 317

Lockman, F. J. 1984, ApJ, 283, 90

Mayer-Hasselwander, H., et al. 1980, Ann. NY Acad. Sci., 336, Ninth Texas Symp. on Relat. Astrophys., 211

Mayer-Hasselwander, H., et al. 1982, A\&A, 105, 164
Moskalenko, I. V., \& Strong, A. W. 1999, ApL, 38, 445

Moskalenko, I. V., \& Strong, A. W. 2000, Ap\&SS, 272, 247

Pohl, M., \& Esposito, J. A. 1998, ApJ, 507, 327

Porter, T. A., \& Protheroe, R. J. 1997, J. Phys. G, 23, 1765

Pühlhofer, G., et al. 1997, Astropart. Phys., 8, 101

Pühlhofer, G, et al. 1999, Proc. 26th ICRC, 77

Reynolds, P. T., et al. 1993, ApJ, 404, 206

Safi-Harb, S., \& Ögelmann, H. 1997, ApJ, 483, 868

Sreekumar, P., et al. 1998, ApJ, 494, 523

Strong, A. W., et al. 1988, A\&A, 207, 1

Strong, A. W., Moskalenko, I. V., \& Reimer, O. 2000, ApJ, 537, 763

Tayler, J. H., et al. 1993, ApJS, 88, 529 